Ciencia y Salud, Vol. IV, No. 1, enero-abril, 2020 •ISSN (impreso): 2613-8816 • ISSN (en línea): 2613-8824

DOI: https://doi.org/10.22206/cysa.2020.v4i1.pp17-26

\title{
DIABETES MELLITUS TIPO 2: UNA PROBLEMÁTICA ACTUAL DE SALUD EN LA POBLACIÓN PEDIÁTRICA
}

\author{
Type 2 diabetes mellitus: A current health problem in the pediatric \\ population
}

\section{Francisco Carvajal Martínez $z^{\mathrm{a}}$, Yadenys Bioti Torres ${ }^{\mathrm{B}}$ y Mónica Carvajal Aballe}

Recibido: 2 de septiembre, 2019 • Aprobado: 3 de diciembre, 2019

Cómo citar: Carvajal Martínez F, Bioti Torres Y, Carvajal Aballe M. Diabetes mellitus tipo 2: una problemática actual de salud en la población pediátrica. cysa [Internet]. 3 de marzo de 2020 [citado 4 de marzo de 2020];4(1):17-6. Disponible en: https://revistas.intec. edu.do/index.php/cisa/article/view/1670

\section{Resumen}

La diabetes mellitus tipo 2 resulta de la combinación de resistencia a la insulina junto con inadecuada secreción de la misma. Su etiología es heterogénea, implicándose en los mismos, factores genéticos y ambientales. Su incremento ha sido significativo en las últimas décadas lo cual ha estado aparejado al incremento de la obesidad. Pudiendo existir tres formas de presentación: asintomática, sintomática y cetosis o cetoacidosis.

El desarrollo acelerado de las complicaciones está dado por una disminución más rápida y progresiva de la función de las células B que caracteriza esta enfermedad en la edad pediátrica.

El tratamiento incluye cambios del estilo de vida, el autocontrol de la diabetes y el tratamiento farmacológico, siendo esencial involucrar a toda la familia en el manejo de este grupo de pacientes. Solo la metformina y la insulina han sido aprobadas para su utilización en este grupo de edad.

\footnotetext{
a Profesor titular y consultante, investigador titular y de mérito. Departamento de Endocrinología Pediátrica, Instituto Nacional de Endocrinología, La Habana, Cuba. Correo-e: Endoped@infomed.sld.cu
}

\begin{abstract}
Type 2 diabetes mellitus results from the combination of insulin resistance along with inadequate secretion of it. Its etiology is heterogeneous, involving genetic and environmental factors. Its increase has been significant in recent decades which has been coupled with the increase in obesity. There may be three forms of presentation: asymptomatic, symptomatic and ketosis or ketoacidosis.

The accelerated development of complications is due to a more rapid and progressive decrease in the function of the $\mathrm{B}$ cells that characterizes this disease in the pediatric age.

The treatment includes lifestyle changes, diabetes self-control and drug treatment, being essential to involve the whole family in the management of this group of patients. Only metformin and insulin have been approved for use in this age group.
\end{abstract}

\footnotetext{
b Profesor Asistente. Especialista de Primer Grado en Endocrinología. Especialista de Primer Grado en Medicina General Integral, Departamento de Endocrinología Pediátrica, La Habana, Cuba

${ }^{c}$ Especialista de Primer Grado en Oncología Médica, Especialista de Primer Grado en Medicina General Integral, Máster en Enfermedades Infecciosas, Instituto Nacional de Oncología, La Habana, Cuba
} 
La prevención es la piedra angular para frenar la oleada de DM2 en niños y adolescentes a nivel internacional. Se basa en la identificación de los individuos en riesgo, diagnóstico precoz de estados prediabéticos y la educación, dirigida a la familia y a la comunidad.

Palabras claves: diabetes mellitus tipo 2; obesidad; niño; adolescente.

\section{Introducción}

En las últimas décadas se ha observado un aumento significativo de la diabetes mellitus tipo 2 (DM2) en las edades pediátricas, lo cual se ha asociado al incremento de la obesidad infantil. ${ }^{1}$

Aunque clásicamente la DM 2 ha sido considerada un síndrome de personas adultas, en el año 1916 ya Reisman había reconocido que en algunos niños con diabetes mellitus las manifestaciones clínicas eran leves y tenían una progresión lenta, similar a lo que ocurre en los adultos. ${ }^{2}$ No obstante, la DM2 no fue reconocida en edades pediátricas hasta principio de los años setenta.

La DM2 de la infancia y la adolescencia, se define por criterios diagnósticos similares a los utilizados en población adulta. Resulta de la combinación de resistencia a la insulina junto con inadecuada secreción de la misma. Su etiología es heterogénea, y los factores ambientales, sociales y de comportamiento son los que desenmascaran la susceptibilidad genética. ${ }^{3}$

La evidencia sugiere que la diabetes tipo 2 en la juventud es diferente no solo de la diabetes tipo 1 , sino también de la diabetes tipo 2 en adultos, dado por una disminución más rápida y progresiva de la función de las células $\mathrm{B}$ y un desarrollo acelerado de las complicaciones de la diabetes. ${ }^{4,5}$
Prevention is the cornerstone to curb the surge of DM2 in children and adolescents internationally. It is based on the identification of individuals at risk, early diagnosis of prediabetic states and education, aimed at the family and the community.

Keywords: Type 2 diabetes mellitus; obesity; kid; Teen.

\section{Epidemiología}

La DM2 tiene un pico de incidencia entre los 12 y los 16 años, que coincide con estadios medios o finales de la pubertad, pueden tener cetosis e incluso cetoacidosis, pérdida de peso leve y en el $90 \%$ de los casos se observa acantosis nigricans, que indica hiperinsulinismo de larga evolución. ${ }^{6}$

Aunque la diabetes mellitus tipo 1 (DM1) sigue siendo la más frecuente en la edad pediátrica en numerosos países, la DM2 ha llegado a superarla en algunos como Japón (3 por 100.000 jóvenes) y Taiwán $(6,5$ por 100.000$) .^{7}$

En la mayoría de los estudios relacionados con la DM2 en niños y jóvenes se ha confirmado una significativa asociación con el incremento de la obesidad en estas edades. ${ }^{8}$

Estimaciones recientes sugieren que al menos la mitad de la población pediátrica con diabetes pertenece al tipo 2, ${ }^{9}$ con una incidencia en los Estados Unidos de 5000 casos nuevos por año. ${ }^{10}$ Los Centros para el Control y Prevención de Enfermedades publicaron proyecciones sobre la prevalencia de la diabetes tipo 2 utilizando la base de datos SEARCH, donde suponen un incremento de un 2,3\% anual, y una prevalencia en el grupo de menores de 20 años de edad que se cuadruplicará en 40 años. ${ }^{11,12}$ 
En la actualidad, la incidencia de diabetes tipo 2 en Estados Unidos ha pasado del 8 al $45 \%$ y en determinadas etnias llega hasta el $94 \% .^{6}$ La población más estudiada es la de los indios Pima con la prevalencia más alta.

En Europa, la incidencia de DM2 sigue siendo baja, del orden del 0,5 al $1 \%,{ }^{13}$ encontrándose hasta un $2,4 \%$ en población adolescente con obesidad grave. ${ }^{14}$ Además de la obesidad, existen otros factores de riesgo importantes como son: el origen étnico, la pubertad (ambos ya señalados), el sexo (más frecuente en mujeres), modo de vida sedentario, antecedentes perinatales (el bajo peso como el aumento de peso), quizás debido a déficit de nutrición o sobre nutrición "en el útero" lo cual pudiera provocar cambios hormonales o metabólicos, ocasionar obesidad, resistencia a la insulina y al final disfunción de las células beta. Se señala que es posible que alrededor del $40 \%$ de los casos de DM2 en nińos indios Pima sea por esta causa. También, es frecuente el antecedente de familiares con diabetes mellitus tipo 2 (puede encontrarse entre el 74-100\%), así como la relación con malos hábitos alimenticios.

La DM2 es más frecuente en indios americanos, negros e hispanos que la población general.

\section{Fisiopatología}

En la DM2 existe un proceso patogénico progresivo que involucra insulinorresistencia (IR) y disfunción de la célula beta pancreática, tanto en los adultos y probablemente en los niños y adolescentes. ${ }^{7} \mathrm{La}$ diabetes tipo 2 es el resultado de la interacción de factores genéticos y ambientales. ${ }^{7,15}$ La susceptibilidad genética es un prerrequisito para que se desarrolle el síndrome. ${ }^{7}$ La importancia del factor genético, poligénico, se basa en la observación de que hijos de pacientes con DM2, delgados y normoglucémicos tienen un metabolismo oxidativo de la glucosa reducido, y una disminución de la síntesis del glucógeno muscular. Hasta la actualidad, solo un pequeño número de genes (involucrados en el desarrollo de la célula $\beta$ y en la síntesis de insulina) se han podido implicar en su riesgo: el gen TCF7L2 (gen 2 del factor de trascripción 7), el gen de la calpaína 10, del canal de $\mathrm{K}$ dependiente de ATP (Kir 6.2), gen del factor hepatonuclear 4, del receptor activado por el perisoma gamma (PPAR $\gamma){ }^{1,16,17}$

Tanto la IR, como el declinar de la célula $\beta$ son fenómenos precoces. Ya en las fases previas a la diabetes, como son la glucemia en ayunas alterada y la tolerancia a la glucosa alterada, hay IR y defectos secretorios de insulina. Cuando aparece la hiperglucemia, aunque se evidencie una marcada hiperinsulinemia, hay un déficit relativo de esta en relación con el valor de glucosa. ${ }^{1}$

En la actualidad, al estudiar la fisiopatología de la DM2 se han identificado un conjunto de mecanismos metabólicos que condicionan la hiperglucemia sostenida presente en el paciente con DM2 (Octeto ominoso $)^{18}$ :

1. Disminución del efecto incretina, impidiendo la secreción prandrial normal de insulina hasta en un $60 \%$.

2. Incremento de la lipólisis.

3. Incremento de la reabsorción tubular renal de glucosa.

4. Disminución de la captación muscular de glucosa.

5. Disfunción de neurotransmisores cerebrales con la consecuente inhibición del centro de la saciedad y con él aumento del apetito.

6. Aumento de la producción hepática de glucosa (Gluconeogénesis).

7. Aumento de la secreción de glucagón por la célula $\alpha$ del páncreas.

8. Disminución paulatina de la secreción de insulina por el páncreas. 
La obesidad visceral presente en los pacientes con diagnóstico de DM2 va acompañada de incremento en los ácidos grasos libres en ayuno y postprandiales. Los ácidos grasos libres que fluyen en la circulación portal provocan acumulación de triglicéridos hepáticos, producción excesiva de lipoproteínas de muy baja densidad (VLDL) y, de manera secundaria, reducción de las concentraciones de lipoproteína de alta densidad (HDL).

Los incrementos en las concentraciones de glucosa se agudizan por la resistencia a la insulina y por la acción del factor de crecimiento de insulina (IGF-I) en el músculo esquelético. ${ }^{12}$

Los mecanismos que pudieran explicar la lipo y glucotoxicidad incluyen los descensos en los transportadores de glucosa 2 de las células beta, así como la expresión de glucocinasa, inhibición de la biosíntesis de insulina, alteraciones en los canales de potasio sensibles a ATP, y una apoptosis acelerada de las células beta. ${ }^{12}$

\section{Historia natural}

Como hemos señalado en la DM2, existe una deficiencia relativa de insulina y una resistencia periférica a la insulina, así como, incremento en la producción de glucosa hepática.

Uno de los factores más frecuentes de riesgo es la pubertad, durante esta etapa se incrementa la resistencia a la insulina lo que ocasiona hiperinsulinemia, posterior a esta etapa los niveles de insulina basal y post prandial disminuyen significativamente. En la pubertad existe incremento transitorio de la hormona de crecimiento, así como, del factor similar a la insulina (IGF-1). También las hormonas esteroideas contribuyen a la resistencia a la insulina.

Un aspecto especial son las adolescentes con síndrome de ovarios poliquísticos, afección que es más frecuente en estas edades, alrededor del $30 \%$ tienen intolerancia a la glucosa y el $4 \%$ presentan DM2.

La historia natural de la DM2 consistiría en: una persona con una base genética asociada a factores de riesgo, lo cual provocaría, en las etapas iniciales de la enfermedad, resistencia a la insulina, ocasionando hiperinsulinismo compensatorio, derivando en agotamiento de las células beta (que es precoz en los niños y adolescentes), lo que se traduce como deficiencia relativa a la insulina. Esto provocaría hiperglucemia post prandial que evolucionaría hacia la intolerancia a la glucosa en ayunas. Posteriormente, al existir la falla significativa de las células beta, aparecería ya, la diabetes mellitus.

Es por eso importante detectar los factores de riesgo para poder prevenir a tiempo la evolución dañina de la enfermedad.

\section{Clínica}

En edades pediátricas pueden existir tres formas de presentación: asintomática, sintomática y hasta en cetosis o cetoacidosis (también reconocida como atípica), esta última con una frecuencia de 5-25\%. Específicamente, en respecto a esto, Calzada ${ }^{19}$ ha señalado una frecuencia hasta del $50 \%$, casi siempre desencadenada por una infección. Por otro lado, desde el punto de vista clínico, en ocasiones es difícil diferenciar entre la DM1 y la DM2, ya que en la primera se puede encontrar aumento de peso (hasta un $24 \%$ ), no obstante, en la DM2 es muy frecuente observar obesidad, también puede existir en ambas, glucosuria. La tipo 1 se presenta igual en los dos sexos, sin embargo, la tipo 2, en ocasiones, es algo más frecuente ( 2 a 1$)$ en las niñas que en los varones. La acantosis nigricans es más frecuente en la tipo 2 que en la tipo 1, es un marcador cutáneo de la resistencia a la insulina, se encuentra entre el 60-90 \% en la DM2 y es más frecuente en pacientes afroamericanos y mexicanos americanos. Si nos 
apoyamos en los diferentes estudios, estos nos pueden demostrar que la secreción de insulina está disminuida o ausente en la tipo 1 mientras en la tipo 2 puede ser variable.

Los niveles de péptido $\mathrm{C}$ y de insulina están elevados al momento de diagnóstico en la tipo 2, aunque pueden disminuir debido a la glucotoxicidad. Por lo que se ha seńalado que las concentraciones de ambos, o sea, péptido $\mathrm{C}$ e insulina, no pueden ser en ocasiones elementos diferenciadores entre ambos tipos de diabetes. También se ha descrito pacientes pediátricos con DM2 (74\%) marcadores autoinmunes de los islotes pancreáticos.

La forma sintomática pudiera presentar al igual que la diabetes tipo 1 poliuria, polidipsia, fatiga y polifagia. En estos la clínica es debido a la hiperglucemia.

\section{Diagnóstico}

El diagnóstico debe estar basado en la evaluación de los datos clínicos, así como de complementarios específicos: péptido C, insulina en ayunas, marcadores autoinmunitarios (anticuerpos contra los islotes, anticuerpos contra descarboxilasa de ácido glutámico) etc.

Los estudios recomendados son: glucemia en ayunas y post prandial de dos horas, así como hemoglobina glucosilada al inicio y después cada 3-4 meses. También se debe evaluar el perfil lipídico, y la presencia o no de micro albuminuria. La Sociedad Americana de Diabetes (ADA) recomienda que, si el paciente tiene 10 o más años de edad, o se encuentra en etapa puberal y, además, es obeso o con sobrepeso y se está valorando la posibilidad de una DM2 o prediabetes, se realice prueba de tolerancia a la glucosa midiendo glucemia basal y a las 2 horas. $\mathrm{Si}$ los resultados son normales se deben repetir alrededor de los 3 años de intervalo, o más frecuente si incrementan el peso corporal (IMC). ${ }^{10}$

\section{Complicaciones o comorbilidades}

Se pueden observar numerosas en el paciente con DM2 sobre todo obesos o con sobrepeso, entre ella podemos citar: nefropatía, hipertensión arterial y alteración de la función cardiovascular, neuropatía, retinopatía, hígado graso no alcohólico, apnea de sueño, síndrome de ovarios poliquísticos, dislipidemia, factores psicosociales, otras. Analizaremos algunas de ellas ${ }^{10}$ :

La hipertensión arterial primaria o esencial es la causa más común de hipertensión en adolescentes con sobrepeso, incrementa el riesgo de complicaciones vasculares y se ha encontrado en adolescentes con DM2 en un 17-32\%.

La dislipidemia en adolescentes con DM2 oscila entre el 4-33\%, se debe evaluar al diagnóstico de la diabetes y post control glucémico, los valores lipídicos óptimos serían: LDL-C $<100 \mathrm{mg} / \mathrm{dl}(2.6 \mathrm{mmol} / \mathrm{l})$, HDL-C > $35 \mathrm{mg} / \mathrm{dl}(0.905 \mathrm{mmol} / \mathrm{l})$ y triglicéridos $<150 \mathrm{mg} / \mathrm{dl}(1.7 \mathrm{mmol} / \mathrm{l})$.

El hígado graso no alcohólico, es la alteración hepática más frecuente en la infancia, no debemos olvidar que puede evolucionar de una esteatosis hepática hacia una esteatohepatitis, posterior a una esteatofibrosis y finalizar en una cirrosis hepática. De ahí la importancia, de la evaluación de la función hepática en todo paciente con DM2 con o sin sobrepeso-obesidad.

Por otro lado, la micro albuminuria (MCA) se presenta entre el 14-22\% de los adolescentes con DM2 desde el inicio, pudiendo llegar hasta el $60 \%$ durante los siguientes 10 años post diagnóstico. La incidencia de MCA es mayor en la DM2 que en la DM1 en los primeros 10 años de evolución.

A nivel cardiovascular, puede existir envejecimiento prematuro. Se ha descrito incremento del grosor de 
la pared del ventrículo izquierdo y aumento de la rigidez arterial.

En nuestro departamento, se han evaluado 4 pacientes con DM2, de ellos 3 con obesidad y 1 sobrepeso, con edades entre 5 y 19 años, 3 hembras y 1 varón. Se encontró 1 con hipercolesterolemia, 1 hipertrigliceridemia, 1 microalbuminuria positiva, 1 disfunción endotelial (según dilatación arteria braquial) y 1 con grosor íntima media elevada.

\section{Tratamiento}

El tratamiento del niño y adolescente con DM2, incluye cambios del estilo de vida, el autocontrol de la diabetes $y$, de ser necesario, el tratamiento farmacológico.

Consideramos que se debe abarcar de la siguiente forma: no farmacológico y farmacológico.

\section{Tratamiento no farmacológico}

\section{Prevención}

La prevención es la piedra angular y se basa en la educación, esta debe estar dirigida a la familia (desde previo a la concepción), a la comunidad, escuelas, etc. Es importante, que el médico de atención primaria se incorpore a estos aspectos, así como, tratar siempre que se pueda, utilizar los diferentes medios de comunicación: prensa escrita, radial, televisada, publicaciones populares, así como información en las escuelas, etc.

La prevalencia creciente de DM2 en la infancia, obliga a establecer pautas en el tratamiento de esta enfermedad. Estas no solo deberían incluir el diagnóstico precoz de la misma, sino estrategias educativas dirigidas, como hemos señalado, a la prevención de la obesidad, factor de riesgo modificable fundamental en la historia natural de la enfermedad. La educación deberá enfocarse en programas dirigidos a lograr una alimentación saludable, así como la reducción del peso en niños obesos a través del aumento de la actividad física, con ello, la disminución del modo de vida sedentario; en ocasiones, habrá que incorporar terapia del comportamiento. Es necesario conocer las modificaciones del comportamiento, el "apoyo de la familia" es decisivo, deberemos evaluar sus fortalezas y debilidades. Es importante la identificación del liderazgo, así como el contexto cultural, los recursos financieros con que cuenta la familia del nińo y/o adolescente y las relaciones entre los integrantes.

No debemos olvidar que los pacientes en edades pediátricas, de forma general, tienen una actividad física desordenada. Particularmente la enfermedad ocasiona baja mortalidad y en algunas veces pocas complicaciones, por lo que, la familia no se preocupa de una forma significativa. La asesoría en materia de diabetes debe incluir a toda la familia, y la incorporación de todos a las diferentes actividades: ejercicio físico, alimentación, etc.

Las diversas literaturas revisadas coinciden en la importancia de un equipo multidisciplinario en diabetes, para el seguimiento y tratamiento de estos pacientes. Este debe incluir al endocrinólogo pediatra, educadora pediátrica, nutricionista, psicólogo, profesor de educación fisica. ${ }^{2,3}$ El licenciado o profesor de educación física es un elemento fundamental en el grupo multidisciplinario de atención cualificada de estos pacientes, por lo que debe también tener conocimientos adecuados sobre la diabetes mellitus.

Es esencial que toda la familia se involucre en el manejo de estos pacientes, para alcanzar los objetivos terapéuticos, que incluyen: revertir las alteraciones metabólicas, conseguir y mantener cifras de glucemia próximas a la normalidad, mejorar la secreción y la sensibilidad a la insulina, promover hábitos de vida saludables (alimentación y ejercicio), 
mantener un peso corporal adecuado, realizar un cribado periódico y tratamiento, si procede, de las comorbilidades, y prevenir y/o tratar las complicaciones, tanto agudas como crónicas.

\section{Tratamiento no farmacológico}

El tratamiento no farmacológico constituye la piedra angular en el tratamiento de la diabetes, sobre todo en este grupo de edad, sin embargo, muchas de las recomendaciones son adoptadas a partir de los resultados obtenidos en poblaciones adultas. ${ }^{12}$

\section{Dieta}

El objetivo principal es alcanzar el control metabólico, el manejo de la obesidad y la reducción del riesgo cardiovascular, fomentando un modo de vida sano, así como, evitar malos hábitos nutricionales, eliminar el consumo excesivo de alimentos hipercalóricos, de carbohidratos refinados y grasas saturadas.

Se debe aconsejar el consumo de grasas no saturadas, fibra dietética, frutas y vegetales, así como el aporte adecuado de proteínas.

El nutricionista, la dietista y/o la educadora juegan, además del médico, un papel importante es la transmisión de los conocimientos necesarios al paciente y a la familia.

Es relevante que en la confección de los planes de alimentación participen el paciente y los familiares, junto a la dietista y/o nutricionista, la enfermera, el educador, el trabajador social, el psicólogo y el médico. Esta debe ser individualizada y dirigida a toda la familia en función de la edad, sexo, actividad física, eventos fisiológicos, teniendo en cuenta su poder adquisitivo familiar y las preferencias culturales. ${ }^{2,3}$

\section{Ejercicio físico}

Es conocido que la actividad física mejora la sensibilidad a la insulina, tiene impacto en la disminución del peso corporal, en la mejoría de la capacidad física y en la resistencia cardiopulmonar.

Es importante disminuir 60 minutos/día enfrente de una pantalla (televisión, computadoras, videos, etc.) y aumentar 60-90 minutos de actividad física.

Se recomienda ejercicios aeróbicos, en forma de juegos, en niños pequeños, y en los mayorcitos, durante una clase de educación física de aproximadamente 45 minutos. En ocasiones, es preferible, si el paciente es extremadamente obeso, caminar. ${ }^{20}$

En nuestro país, se ha venido desarrollando un programa de actividades grupales y de convivencia los cuales son de gran ayuda en materia de educación, para la obtención de las metas trazadas en los niños y adolescentes diabéticos.

\section{Tratamiento farmacológico}

El tratamiento inicial del niño y adolescente con diabetes que se asocia a obesidad debe tener en consideración que en muchas ocasiones el tipo de diabetes es incierto en las primeras semanas de tratamiento. Por lo tanto, la terapia inicial debe abordar la hiperglucemia y los trastornos metabólicos asociados, independientemente del tipo de diabetes final, con un ajuste de la terapia una vez que se haya establecido la compensación metabólica y se disponga de información posterior, como los resultados de los auto anticuerpos de los islotes, etc. ${ }^{8}$

Los objetivos glucémicos deberán ser individualizados. La $\mathrm{ADA}^{10}$ plantea como objetivo de niveles de hemoglobina glucosilada (HbA1c) razonable para la mayoría de los adolescentes con diabetes tipo 2 tratados solo con agentes orales de $7 \%$ 
(53 mmol/mol). Mientras que objetivos más estrictos de la misma (HbA1c $6.5 \%$ ) pudiera considerarse en pacientes con poco tiempo de evolución de la diabetes, menor grado de disfunción de las células B y los pacientes tratados con estilo de vida o solo metformina, que hayan logrado una mejoría significativa del peso.

El objetivo de una HbA1c más baja en los jóvenes con diabetes tipo 2 en comparación con los recomendados en la diabetes tipo 1 se justifica por un menor riesgo de hipoglucemia y un mayor riesgo de complicaciones. ${ }^{10}$

Las opciones farmacológicas actuales para la DM2 al diagnóstico, se encuentra limitado a dos medicamentos aprobados por la Food and Drug Administration (FDA) y la American Pediatric Association: la metformina y la insulina, ${ }^{2}$ aunque la prescripción de hipoglucemiantes en el grupo que nos ocupa es frecuente, sin embargo, la evidencia a favor de cualquiera de dichas modalidades fármaco terapéuticas es muy limitada. ${ }^{12}$

Se deberá considerar la terapia farmacológica en aquellos pacientes en los que tras 3-6 meses de medidas no farmacológicas no se consiga un control adecuado o, desde el inicio, en pacientes sintomáticos, sobre todo cuando exista cetosis o cetoacidosis diabética. $^{3}$

El tratamiento insulínico estará indicado en las presentaciones con cetoacidosis o cetosis, cuando la distinción entre diabetes tipo 1 y diabetes tipo 2 no está clara y en pacientes que tienen concentraciones aleatorias de glucosa en sangre $250 \mathrm{mg} / \mathrm{dL}$ $(13.9 \mathrm{mmol} / \mathrm{l}) \mathrm{y} / \mathrm{o} \mathrm{HbA} 1 \mathrm{c} 8.5 \%(69 \mathrm{mmol} / \mathrm{mol}) .{ }^{10}$ También se indicará cuando no se cumple el objetivo glucémico con el uso de metformina solamente, o si hay intolerancia a la misma, o insuficiencia renal, o hepática. ${ }^{10}$
Cuando no se requiere tratamiento con insulina, se recomienda el inicio de metformina, ${ }^{8}$ asociados a los cambios en el estilo de vida. El estudio "Opciones de tratamiento para la diabetes tipo 2 en adolescentes y jóvenes" (TODAY) es el único ensayo que combina el estilo de vida y la terapia con metformina en jóvenes con diabetes tipo 2 , sin embargo, en el mismo se encontró que la combinación de estos no funcionó mejor que el uso de metformina sola para lograr un control glucémico duradero. ${ }^{10}$

Se considera la metformina eficaz y segura, con beneficio adicional por ligera pérdida de peso o estabilidad de este, no ocasiona hipoglucemia, mejora el perfil lipídico y actúa sobre la resistencia a la insulina. Se debe comenzar con dosis bajas $(500 \mathrm{mg}$. 2 veces al día, a mitad de la ingesta) que se van incrementando progresivamente según tolerancia y efectos obtenidos, hasta una dosis máxima de 2,000 mg. /día. La edad de inicio de tratamiento se ha seńalado que puede ser desde los 4 años de edad. ${ }^{19}$

La metformina actúa sobre los receptores de insulina en la grasa, músculo e hígado, disminuye la producción de glucosa hepática, la gluconeogénesis y aumenta la absorción de glucosa estimulada por insulina. Se debe tener cuidado por los posibles efectos secundarios: dolor abdominal (25\%), náuseas y vómitos (17\%), así como diarreas y cefaleas. Es recomendable ingerir conjuntamente con complejo vitamínico, ya que puede disminuir la absorción de vitamina B12 y/o ácido fólico. No debe ser utilizada si el paciente presenta cetosis, insuficiencia renal, alteraciones hepáticas, cardiaca o ha recibido contrastes iodados.

La cirugía bariática o cirugía metabólica se ha realizado cada vez más en adolescentes con obesidad. Como una indicación para esta cirugía, se incluye, generalmente, un IMC de $35 \mathrm{~kg} / \mathrm{m} 2$ con comorbilidades o un IMC de $40 \mathrm{~kg} / \mathrm{m} 2$ con o sin comorbilidades. ${ }^{10,21}$ 
Los adolescentes experimentan grados similares de pérdida de peso, remisión de la diabetes y mejoría de los factores de riesgo cardiometabólicos durante al menos tres años después de la cirugía. Sin embargo, ningún ensayo aleatorizado ha comparado la efectividad y la seguridad de la cirugía con las opciones de tratamiento convencionales en adolescentes.

\section{Aspectos psicosociales}

Como hemos señalado, es necesario conocer las características de la familia del niño y/o adolescente con DM2, conocer su contexto psicosocial y sociocultural, sus barreras y dificultades económicas entre otros aspectos a considerar.

Debemos recordar, que el niño/adolescente con DM2 tiene una carga cognoscitiva negativa en relación con su enfermedad, por lo que el equipo multidisciplinario debe actuar en ese sentido para minimizar las dificultades y que el paciente/familia sea un apoyo más para lograr los objetivos planificados.

Hay que orientar y vigilar principalmente en el adolescente, los trastornos depresivos, el uso de bebidas alcohólicas, el tabaquismo, así como otras situaciones como las drogas y embarazo precoz (sin orientación médica y frecuentemente no deseado).

Por otro lado, la transición de la atención pediátrica a los servicios especializados de adultos, debe ser preparada con cuidado, informada con suficiente tiempo a los pacientes y familiares, recomendado al menos un año antes de su realización.

\section{Bibliografía}

1. Ros P, Barrio R. Diabetes tipo 2 en la infancia y adolescencia. An Pediatr Contin. 2009;7(3): 12735. DOI: $10.1016 / S 1696-2818(09) 71116-3$

2. Licea ME, Bustamante M, Lemane M. Diabetes tipo 2 en nińos y adolescentes: aspectos clíni- co-epidemiológicos, patogénicos y terapéuticos. Rev Cubana Endocrinol. 2018;19(1).

3. Grey M, Schreiner B, Pyle L. Development of a diabetes program for youth with type 2 diabetes. Diabetes Educ 2009;35: 108-6.

4. Arslanian S, Bacha F, Grey M, Marcus MD, White NH, Zeitler P. Evaluation and management of youth-onset type 2 diabetes: a position statement by the American Diabetes Association. Diabetes Care 2018;41: 2648-68.

5. Copeland KC, Zeitler P, Geffner M, et al.; TODAY Study Group. Characteristics of adolescents and youth with recent-onset type 2 diabetes: the TODAY cohort at baseline. J Clin Endocrinol Metab 2011;96:159-67.

6. Amaya M. ${ }^{a}$ J., Colino E, López-Capapé M, Alonso M, Barrio R. Diabetes mellitus tipo 2 en la edad pediátrica. An Pediatr (Barc) 2005; 62(2):174-7.

7. Barrio R, Ros P. Diabetes tipo 2 en población pediátrica espańola: cifras, pronóstico y posibilidades terapéuticas. Av Diabetol. 2013;29 (2): 27-35.

8. Dabelea D, Mayer-Davis EJ, Saydah $S$ et al. SEARCH for Diabetes in Youth Study. Prevalence of type 1 and type 2 diabetes among children and adolescents from 2001 to 2009. JAMA 2014;311: 1778-86.

9. Frenk Baron P,Márquez E. Diabetes mellitus tipo 2 en niños y adolescentes. Med Int Mex 2010;26(1): 36-47.

10. Standards of Medical Care in Diabetes-2019. Children and Adolescents Type 1 Diabetes Type 2 Diabetes, Transition From Pediatric to Adult Care. Diabetes Care. January 2019;42: Supplement 1. 
11. Imperatore G, Boyle JP, Thompson TJ, et al. SEARCH for Diabetes in Youth Study Group. Projections of type 1 and type 2 diabetes burden in the U.S. population aged 20 years through 2050: dynamic modeling of incidence, mortality, and population growth. Diabetes Care. 2012; 35: 2515-20.

12. Pettitt DJ, Talton J, Dabelea D, et al.; SEARCH for Diabetes in Youth Study Group. Prevalence of diabetes in U.S. youth in 2009: The SEARCH for Diabetes in Youth Study. Diabetes Care 2014;37:402-8.

13. Vivian EM. Type 2 diabetes in children and adolescents. The next epidemic? Curr Med Res Opin. 2006;22: 297-306-17.

14. Felszeghy E, Juhasz E, Kaposzta R, Ilyes I. Alterations of glucoregulation in childhood obesity association with insulinresistance and hyperinsulinemia. J Pediatr Endocrinol Metab.2008;2: 847-53.

15. Medina N. Diabetes mellitus tipo 2 en Pediatría. La importancia del cribado y tratamiento precoz. Evid Pediatr. 2017;13: 44.

16. Gungor N, Bacha F, Saad R, Janosky J, Arslanian $S$. Youth type 2 diabetes, Insulin resistance, b-cell failure, or both? Diabetes Care. 2005;28: 638-44. Medline
17. Prentki M, Nolan Ch J. Islet b cell failure in type 2 diabetes. JClin Invest. 2006;116: 1802-12. Medline.

18. De Fronzo RA. From the Triumvirate to the Ominous Octet: A new paradigm for the Treatment of Type 2 Diabetes Mellitus. Diabetes. 2009;58(4): 773-95.

19. Calzada R. Comunicación personal. 1er. Congreso Centroamericano y del Caribe de Endocrinología Pediátrica. 2019. Honduras.

20. Carvajal F. Diabetes Mellitus y Ejercicio Físico. (2da ed.). Cuba: Editorial Pueblo y Educación; 1999.

21. Inge $\mathrm{TH}$, Courcoulas $\mathrm{AP}$, Jenkins $\mathrm{TM}$ et al. Teen-LABS Consortium. Weigh loss and health status 3 years after bariatric surgery in adolescents. N Engl J Med 2016;374: 113-23.

22. Levitt Katz LE, Swami S, Abraham M et al. Neuropsychiatric disorders at the presentation of type 2 diabetes mellitus in children. Pediatr Diabetes 2005;6: 84-9 